\title{
Magnetic triggering of ELMs in TCV
}

\author{
A.W. Degeling ${ }^{1}$, Y. R. Martin ${ }^{1}$, J. B. Lister ${ }^{1}$, L. Villard ${ }^{1}$, V. N. Dokouka ${ }^{3}$, \\ V. E. Lukash ${ }^{2}$ and R. R. Khayrutdinov ${ }^{3}$ \\ ${ }^{1}$ Centre de Recherches en Physique des Plasmas, Association Euratom-Confédération Suisse \\ Ecole Polytechnique Fédérale de Lausanne, CH-1015 Lausanne, Switzerland \\ ${ }^{2}$ RRC Kurchatov Institute, Moscow, Russia \\ ${ }^{3}$ TRINITI, Troitsk, Moscow Region, Russia
}

\begin{abstract}
Experiments on the TCV tokamak have shown that rapid vertical movement of diverted ELMy H-mode plasmas can affect the time sequence of Edge Localised Modes. The effect is attributed to the induction of an edge current during the movement of the plasma column in the spatially inhomogeneous vacuum field of a single null configuration. In TCV the fast vertical movement is provoked by the positional control coils inside the vacuum vessel, however it is argued that a similar effect might be produced in larger devices only using poloidal field coils external to the vessel. A simple model, which includes plausible elements of the dynamical behaviour of the edge pressure gradient and edge current, which together dictate the MHD stability of the discharge against edge-localised, current-driven modes, is used to reproduce some of the features seen in these experiments.
\end{abstract}

\section{INTRODUCTION}

In tokamak plasmas, the so called high confinement mode (H-mode) is frequently accompanied by bursts of MHD activity and deuterium light $\left(\mathrm{D}_{\alpha}\right)$ emission, known as edge localised modes (ELMs), and H-modes of this type are termed ELMy H-modes [1]. ELMs are momentary breakdowns in the H-mode edge transport barrier, and result in the pulsed release of up to $10 \%$ of the stored plasma energy. The plasma density in an H-mode without ELMs (a so called ELM-free H-mode) is generally non-stationary, and can rise uncontrollably until the plasma disrupts. The cyclic degradation in confinement provided by ELMs therefore has a regulating effect on the particle balance, and allows stationary H-mode operation. ELMs are also useful for removing impurities that build up in the plasma interior, and will aid the removal of He ash in a working reactor. These beneficial properties of ELMs have resulted in the adoption of the ELMy H-mode as the basic operating scenario for ITER. However, ELMs have one serious drawback: the projected energy released per ELM in ITER generates an unacceptable heat load on plasma facing components. For this reason considerable effort 
worldwide has gone into investigating the ELM phenomenon, and ways in which the damaging effect of ELMs could be ameliorated.

An important characteristic of ELMs is that the energy released per ELM scales with the time interval between ELMs $[2,3]$. This suggests that the most damaging ELMs may be avoided if a high ELM frequency $\left(f_{\text {elm }}\right)$ can be maintained. Hence it is interesting to consider ways in which MHD modes that lead to ELM events may be actively destabilised. It has been proposed that exceeding the MHD stability limit for coupled Peeling-Ballooning modes, which is a function of the edge pressure gradient $\left(\nabla p_{\text {edge }}\right)$ and the edge current density $\left(j_{\text {edge }}\right)$, is responsible for triggering ELM events [4,5]. We therefore seek reliable ways to externally perturb these edge parameters in order to trigger ELMs. The motivation for such a study is twofold: firstly to investigate the engineering goal of externally controlling the occurrence of ELMs, and secondly to gain insight into the physics and dynamics of the ELM cycle using a perturbative technique.

This paper reports a recent attempt on TCV to apply electromagnetic perturbations that modulate the edge plasma current density and thereby modify the inter-ELM interval. A similar approach to controlling the occurrence of ELMs has previously been investigated in the Compass-D tokamak [6] using sinusoidal modulation of the ohmic transformer current. This study showed synchronous modulation of the ELM activity, with ELMs occurring in bursts only during the positive induced loop voltage part of the modulation cycle. The modulation frequency, around $50 \mathrm{~Hz}$, was such that many ELMs occurred during one triggered burst. However, it was established during the work that the ELMs could indeed be driven unstable by a positive loop voltage, attributed to the increased edge current destabilising the peeling mode. During this work on Compass-D, the density rise during the otherwise ELM-free phase was reduced by a factor of 5 [6].

The work in this paper differs from the work on Compass-D in two respects. Firstly, modulating the surface loop voltage using the PF coils outside the TCV vacuum vessel using the ohmic transformer coils or the shaping coils did not give interesting results, probably since TCV has a significantly longer vacuum vessel L/R time than Compass-D, although some slight effects were seen on otherwise ELMing H-modes. Secondly, the new triggering method employed on TCV targets specific ELMs, whereas Compass-D showed a change in the general ELMing pattern. 
On JET [7] the effect of ramping the current was also seen to influence the ELMs.

Recently, another approach aimed at triggering individual ELMs has been investigated on ASDEX Upgrade [8] using shallow pellet injection to modify the edge profiles. In this case, individual ELMs were also targeted and regularly triggered.

Cyclic perturbations arriving at the edge from sawtooth crashes in the plasma interior have also been shown to have a synchronising effect on the ELM cycle, with the ELM frequency locking to the sawtooth frequency or its harmonics [9].

The presentation of this paper is as follows. In Section 2 we present the details of the experiments performed on TCV to trigger ELMs using external magnetic perturbations. It is argued that these perturbations couple to the ELM cycle causing synchronisation by modifying the edge current density. Section 3 presents estimations of the effect of the external perturbation to the edge plasma current density using a simple calculation, and also a detailed simulation of the plasma interaction with the vessel and control coils. Comparing our observations with a detailed model of the evolution of ELMs is a challenge beyond the scope of this paper and in Section 4 we limit ourselves to comparing our observations with the behaviour of an illustrative model containing some of the ingredients of a full dynamical ELM model. In Section 5 we consider whether this experiment may be scalable to large scale devices such as ITER, and in Section 6 we present our conclusions.

\section{THE EXPERIMENTS AND RESULTS}

In this set of experiments, a single null diverted, ohmically heated plasma was chosen as the standard condition with an elongation $\kappa=1.74$, triangularity $\delta=0.57$, plasma current $I_{P}=400$ $\mathrm{kA}$, line average density $\mathrm{n}_{\mathrm{e}}=6.5 \times 10^{19} \mathrm{~m}^{-3}$ and ion grad-B drift in the unfavourable direction. Under these conditions a stable ELMy H-mode phase was consistently reproducible with an average $\mathrm{ELM}$ frequency $\mathrm{f}_{\mathrm{elm}} \approx 250 \mathrm{~Hz}$ and typically lasting from $\mathrm{t}=0.4$ to 1.4 seconds, where $\mathrm{t}$ is the time after discharge initiation. The features of these TCV ELMy H-modes have predominantly, though not exclusively Type III characteristics.

Figure 1 shows a cross section of the TCV vacuum vessel and the locations of the various poloidal field (PF) coils. The first and most obvious way to try to modulate jedge was to modulate the voltage applied to the ohmic heating transformer coil (labeled $\mathrm{OH}$ in Figure 1), 
in order to induce perturbations in the surface loop voltage. However the amplitude and frequency of such perturbations are limited by both the coil amplifier voltage and the filtering effect of the stainless steel vacuum vessel. TCV has an unusual advantage of being equipped with a set of coils internal to the vacuum vessel (labeled G coils in Figure 1) that are powered by a "fast" power supply with a bandwidth approaching $10 \mathrm{kHz}$. Two sets of three turns are located inside the top and bottom outer corners of the rectangular vessel, with opposing toroidal current directions to produce a purely radial field on the vessel midplane.

Single null divertor plasmas are by nature up-down asymmetric. In normal TCV operation the discharge is usually shifted with respect to the vessel midplane. Driving the G coils therefore has two direct effects: 1) a net surface loop voltage is induced when the plasma is closer to one set of G coils than the other; 2) the vertical motion of the plasma caused by the time varying $\mathrm{G}$ coil currents and the induced vessel image currents will generate an edge current because of the up-down asymmetry of the vacuum field.

The G coils are used for vertical stabilisation of the plasma position. The drive signal to the coil power supplies is provided by a feedback control system based on magnetic measurements. A series of pulses of $1 \mathrm{~ms}$ duration, with a variable delay between pulses, was injected into the vertical stabilisation feedback loop. This perturbation to the feedback loop resulted in spike - like pulses in the G coil current up to $2 \mathrm{kA}$ (peak to peak), illustrated in Figure 2. The vertical position stabilisation is a closed loop system that reacts to the perturbation as a result of the movement of the plasma current itself. The closed loop response to the perturbation provides a complex signal on all of the coil voltage, coil current and plasma position signals. Figure 2 defines the inter-pulse interval $\Delta \mathrm{t}_{\mathrm{D}}$, the inter-ELM interval $\Delta \mathrm{t}_{\mathrm{elm}}$ and the time delay from each pulse to the next ELM $\Delta \mathrm{t}_{\mathrm{p} \rightarrow \mathrm{elm}}$. Figure 2 also shows the effect of an ELM on the closed loop response. The ELM causes a vertical jump corresponding to the change of poloidal beta and inductance in the inhomogeneous vacuum field.

Figure 3 (left) shows an example of the first of a series of three TCV discharges. In discharge \#20332 a constant amplitude pulse train was applied to the $G$ coils with a delay between pulses decreasing from $\Delta t_{D}=7$ to 3 milliseconds. The panels show the $\mathrm{D}_{\alpha}$ timeseries (indicating the ELM activity), the G coil current, and the series of $\Delta \mathrm{t}_{\mathrm{elm}}, \Delta \mathrm{t}_{\mathrm{D}}$ and $\Delta \mathrm{t}_{\mathrm{p} \rightarrow \mathrm{elm}}$. The perturbed current in the $\mathrm{G}$ coils is of similar magnitude to the control system response to each ELM event. In this case, the ELMs appear to be unaffected by the applied perturbation. In particular, there does not appear to be any relationship between the ELM intervals and the 
interval between pulses as it is swept during the discharge (indicated by the dashed line in the figure), nor is there any trend in the time delay from each pulse to the next ELM. The only exception is between $\mathrm{t}=1$ and 1.1 seconds, when the driver period $\Delta \mathrm{t}_{\mathrm{D}}$ crosses the ELM period $\Delta \mathrm{t}_{\mathrm{elm}}$, causing $\Delta \mathrm{t}_{\mathrm{p} \rightarrow \mathrm{elm}}$ to vary slowly over this time.

Figure 3(right) shows the second discharge (\#20333) in which the input perturbation was increased by a factor of 4 , resulting in current spikes over $1 \mathrm{kA}$ in the $\mathrm{G}$ coils. In this case $\Delta \mathrm{t}_{\mathrm{elm}}$ increases well above its average unperturbed value when $\Delta \mathrm{t}_{\mathrm{D}}$ is initially high, and decreases gradually below its unperturbed value as $\Delta t_{D}$ is reduced. There are several instances where the ELM period clearly tracks the driving pulse intervals for significant periods. In particular, locking between $\Delta \mathrm{t}_{\mathrm{D}}$ and $\Delta \mathrm{t}_{\text {elm }}$ is observed over the following time ranges in seconds: $0.6-0.73,0.74-0.79,0.81-0.86,0.88-0.94$ and $0.98-1.11$. This entrainment is momentarily broken a number of times in between these intervals, resulting in wide excursions of the ELM period from its local average value.

It is important to note that the general scaling of ELM amplitude with $\Delta \mathrm{t}_{\text {elm }}$ is preserved in the presence of the external perturbations, regardless of whether or not the ELMs are entrained. This is demonstrated by plotting the ELM amplitude against $\Delta \mathrm{t}_{\text {elm }}$, as shown in Figure 4 for discharge \#20333. As expected, the ELMs occurring in the absence of the perturbation signal (dots) mark out a linear trend in this figure. Small, high frequency ELMs that give rise to the large range in $\Delta \mathrm{t}_{\mathrm{elm}}$ in this data occurred during non-stationary phases at the beginning and end of the H-mode. The ELMs that occurred as the external driver signal was swept from $\Delta \mathrm{t}_{\mathrm{D}}$ $=7$ to $3 \mathrm{~ms}$ (squares) show the same linear scaling of amplitude with $\Delta \mathrm{t}_{\mathrm{elm}}$ as that found in the absence of the perturbation. This suggests that if magnetic triggering can reliably reduce the maximum inter-ELM period, then the largest and most damaging ELM amplitudes should be avoided.

A more illustrative way to observe correlations between the ELMs and the external driver is to plot the relative phase, defined for each ELM by

$$
\phi=2 \pi \frac{\Delta \mathrm{t}_{\mathrm{p} \rightarrow \mathrm{elm}}}{\Delta \mathrm{t}_{\mathrm{D}}}
$$

Figure 5 shows the variation of $\phi$ with the $2 \pi$ wrapping in phase removed, against the drive frequency $\left(f_{D}=\Delta t_{D}^{-1}\right)$ for discharges \#20332 and \#20333. In the absence of coupling the 
phase smoothly ramps at a rate defined by the difference in frequency between the perturbation and the ELMs. This is illustrated by the dotted line in this figure, which was produced by taking a linear best fit of $\Delta \mathrm{t}_{\mathrm{elm}}$ from a preceding discharge with no perturbation to calculate the expected variation of the phase. The phase of the ELMs in discharge \#20332 (solid line connected by points) clearly follows this curve, confirming the absence of coupling in this case. The evolution of $\phi$ in discharge \#20333 contrasts strongly, showing instead a staircase pattern, as the ELMs maintain a stationary phase with respect to the perturbation for extended periods, before abruptly slipping by $2 \pi$ radians. These episodes of phase slipping correspond to the excursions in the ELM period in Figure 3c).

The first three rows in Figure 6 show a detailed view of the closed loop response of the G coil voltage, current and the plasma position to the input perturbations, for three discharges: \#20333, \#20334 and \#22768. In each case, the trace was produced by phase coherent averaging with respect to the perturbation times over a time interval from $\Delta \mathrm{t}=-2 \mathrm{~ms}$ to $\Delta \mathrm{t}=$ $+4 \mathrm{~ms}$, where $\Delta \mathrm{t}=0$ corresponds to the onset time of each perturbation. A time window of at least 0.4 seconds was chosen for each discharge, which included a period where synchronisation between the ELMs and the perturbation was observed and avoided the time at which $\Delta \mathrm{t}_{\mathrm{D}}$ crossed the unperturbed value of $\Delta \mathrm{t}_{\mathrm{elm}}$. The last row in Figure 6 shows the observed distribution of $\Delta \mathrm{t}_{\mathrm{p} \rightarrow \mathrm{elm}}$ from $\Delta \mathrm{t}=-2 \mathrm{~ms}$ to $+4 \mathrm{~ms}$ for each discharge, which was taken from the ELM occurrences over the same fraction of the discharge. Note that discharge \#20332 (not shown) has a flat distribution.

Discharge \#20334 repeated conditions of \#20333, with a perturbation of the same amplitude as \#20333, but with the polarity of the perturbation reversed. Entrainment similar to the previous discharge was observed in this case, although over a reduced range in $f_{D}$. Discharge \#22768 inverted the position of the plasma in the TCV vessel, and used a perturbation with the same sign as \#20333. Figure 6 shows that the positive then negative perturbation caused a downward, then upward shift in the plasma position $(\Delta z)$ in discharges \#20333 and \#22768, while the opposite polarity perturbation in discharge \#20334 caused an upward then downward shift in the plasma position. The distributions of $\Delta \mathrm{t}_{\mathrm{p} \rightarrow \mathrm{elm}}$ in each discharge show a single significant peak, indicating the coupling effect between the ELMs and the perturbation, however the timing of the peak with respect to the perturbation time varies. For discharges \#20334 and \#22768 the peak coincides with the (respectively negative or positive) peak in $\mathrm{I}_{\mathrm{G}}$ and the leading edge of the excursion in $\Delta z$, whereas for discharge \#20333, the peak occurs 
well after the peak in $\mathrm{I}_{\mathrm{G}}$, and coincides with the trailing edge of the excursion in $\Delta \mathrm{z}$. The consistent feature between all three discharges is that the ELMs are most likely to occur when the plasma moves in a direction that gives rise to an increase in the edge current density - that is upwards when the plasma is in a single-null lower configuration, and downwards when in a single-null upper configuration.

The sign of the edge current perturbation due to the plasma movement can be argued simply. The current in the poloidal field coils responsible for creating the X-point and the up-down asymmetry of the vacuum magnetic flux pattern has the same sign as the plasma current. If the plasma moves towards this current, Lenz's law implies a negative surface voltage and hence a negative edge current will be induced. Consequently, an upward vertical movement in a lower single-null configuration will induce a positive edge current. This strongly suggests that the dominant effect of the $\mathrm{G}$ coil driver signal is to perturb the edge current by displacing the plasma vertically. Increasing the edge surface current has a destabilising effect on the edge mode stability [4,5], consistent with provoking the destabilisation of the modes responsible for causing an ELM.

Figure 7 compares details of the frequency entrainment with the external drive for discharges \#20333 and \#20334. The synchronising effect is also visible in the evolution of the phase with drive frequency for \#20334, which again shows stationary intervals interspersed with rapid ramps through $2 \pi$ between steps. Dotted lines in the figure show the phase variation expected if no coupling existed between the ELMs and the driver during episodes where phase slipping occurs for discharge \#20333. These curves were produced by simply shifting the "no coupling" case from Figure 5 vertically to overlay each phase slipping occurrence. In each case, the phase slipping in \#20333 is faster than with no coupling. This implies that during these times the ELM dynamics are still affected by the perturbation even though the ELMs have lost synchronisation. In other words, the synchronising effect of the perturbation does not appear to be simply the provision of a discrete trigger event, which either leads directly to an ELM, or has no effect on the ELM dynamics if it does not. This is also indicated in the variation of $\Delta t_{\mathrm{elm}}$ during the phase slipping episodes, shown in Figure 3c). If coupling between the ELMs and the perturbation was momentarily lost during one of these intervals, then $\Delta t_{\text {elm }}$ would be expected to simply return from $\Delta t_{D}$ to a value close to the unperturbed average value $\left(\Delta \mathrm{t}_{\text {elm0 } 0}\right)$, such that the size of the excursion in $\Delta \mathrm{t}_{\text {elm }}$ would be roughly $\mid \Delta \mathrm{t}_{\mathrm{D}}$ - 
$\Delta \mathrm{t}_{\text {elm } 0} \mid$. Instead, the excursions in $\Delta \mathrm{t}_{\mathrm{elm}}$ are shown to occur over a much wider range during each phase slipping event.

Another interesting feature of Figure 7 is that many of the phase jumps during the initial part of the $f_{D}$ sweep (140-200 Hz) in these two consecutive discharges occur at similar frequencies. This correlation suggests that the momentary losses in phase lock might not be intermittent or random events, for example caused purely by the presence of noise in the ELM cycle, but may be an intrinsic property of the driven dynamical system.

Additional experiments were carried out with intermediate perturbation amplitudes. Whether or not frequency entrainment took place in each case was ascertained by comparing the unwrapped phase of the ELMs during the perturbation with a control discharge in which no perturbation was applied, as illustrated in Figure 5. In so doing, an estimate was made of the range in driving frequency over which the ELM frequency was affected. This was compared with the peak to peak amplitude of the rate of change in plasma position, which was considered to be the governing parameter, as shown in Figure 8. In this figure, the driving frequency is normalised by the unperturbed ELM frequency during each discharge, which was estimated from the ELM time intervals before and after the perturbation was applied. This figure shows that the range of driving frequency over which the ELMs are affected increases as the perturbation level, estimated by the plasma velocity, is increased.

\section{ESTIMATION OF THE PERTURBED EDGE CURRENT}

An estimation of the perturbation to the edge current density in response to the externally induced vertical displacements may be made as follows.

The surface loop voltage $\mathrm{V}_{\text {surf }}$ is given by

$$
\mathrm{V}_{\text {surf }}=\mathrm{V}_{\mathrm{L}}+\delta \mathrm{V}(\mathrm{t})=-\frac{\mathrm{d}}{\mathrm{dt}}\left\langle\psi_{\text {ext }}\right\rangle=-\frac{\partial}{\partial \mathrm{t}}\left\langle\psi_{\text {ext }}\right\rangle-\left\langle\mathbf{u} \cdot \nabla \psi_{\text {ext }}\right\rangle
$$

where, $\mathrm{V}_{\mathrm{L}}$ and $\delta \mathrm{V}$ are the steady and perturbed surface loop voltages respectively, $\psi_{\text {ext }}$ is the external poloidal flux and the brackets indicate averaging over the last closed flux surface, which moves with a vertical velocity $\mathbf{u}=\mathrm{u}_{\mathrm{z}}(\mathrm{t}) \hat{\mathbf{z}}$ in response to the pulsed currents in the $\mathrm{G}$ coils. The external poloidal flux can be written as $\psi_{\text {ext }}=\psi_{\Omega}+\psi_{\mathrm{G}}$, where $\psi_{\Omega}$ is the poloidal flux from the $\mathrm{E}, \mathrm{F}$ and $\mathrm{OH}$ coil currents in $\mathrm{TCV}$, the rate of change of which gives rise to $\mathrm{V}_{\mathrm{L}}$, and the fluxes $\psi_{\mathrm{G}}$ are contributions from the $\mathrm{G}$ coil currents and their vacuum vessel image 
currents. Assuming that the plasma moves as a rigid body and that $\psi_{\Omega}>>\psi_{\mathrm{G}}$, the level of perturbation to the ohmic component of the edge current density can be estimated by:

$$
\frac{\delta \mathrm{J}}{\mathrm{J}_{\mathrm{o}}}=\frac{\delta \mathrm{V}}{\mathrm{V}_{\mathrm{L}}}=-\frac{1}{\mathrm{~V}_{\mathrm{L}}}\left(\frac{\partial \psi_{\mathrm{G}}}{\partial \mathrm{t}}+\mathrm{u}_{\mathrm{z}}\left\langle\frac{\partial \psi_{\Omega}}{\partial \mathrm{z}}\right\rangle\right)
$$

The average of $\partial \psi_{\Omega} / \partial z$ over the last closed flux surface was carried out numerically for the reconstructed equilibrium of discharge \#20333 (shown by the dashed line in Figure 1) and the peak $u_{z}$ and $V_{L}$ were taken from diagnostic measurements. This yields an estimate for the perturbation caused by the second term in the above equation of up to $10 \%$. That is, we expect variations in the edge current density of up to $10 \%$ of the steady state ohmically driven component to be produced by vertical displacements alone.

The first term, describing the directly induced currents from the $\mathrm{G}$ coils and their vessel images, is much more difficult to estimate simply, and we must instead use the DINA code [10], which numerically solves the free boundary equilibrium problem, including the transport of poloidal flux and thermal energy, assuming instantaneous force balance. DINA was previously equipped to model the full TCV plasma control system, including all PF coils, power supplies and diagnostics and was convincingly validated for stimulation of the external PF coils [11] and for forced VDEs [12]. In these present simulations, the experimental voltage waveforms were applied to the $\mathrm{G}$ coils with all feedback loops closed as in the experiment. A high radial resolution (50 radial grid points) was used to observe the effect of the inward diffusing current perturbation. The full simulation lasted for 0.2 seconds and the perturbations were applied each $10 \mathrm{msec}$. Different step sizes led to similar conclusions. In order to pick out the typical effect of the imposed perturbations, in the presence of sawtoothing and the slow action of the shape control, the perturbation cycles were coherently averaged to increase the signal to noise ratio.

Three discharges already discussed were simulated. Discharges \#20333 and \#20334 provide the reference points with a SNL divertor configuration in the upper part of the TCV vacuum vessel $(\mathrm{z}=+0.23 \mathrm{~m})$ and both signs of voltage perturbations. Discharge \#22678 is a similar equilibrium, but inverted to have a SNU divertor configuration in the lower part of the vessel $(\mathrm{z}=-0.23 \mathrm{~m})$.

The results of these three simulations are shown in Figure 9. The edge current inverts sign with the sign of the perturbation, and has the opposite sign when the equilibrium is inverted. 
This is expected for both the direct induction of an edge current by asymmetry with respect to the $\mathrm{G}$ coil pair and for the speed in the vacuum field gradients. However, a further simulation with a roughly centred equilibrium, in which the first mechanism is smaller, showed similar perturbations to the edge current, suggesting that the dominant mechanism is the poloidal field asymmetry. No attempt was made in these perturbations to model the H-mode edge pedestal. The edge current is significantly modified, by several tens of percent, although this perturbation is reduced to typically $5 \%$ at radial grid points deeper into the plasma. Comparing the time of maximum ELM probability in Figure 6 with the simulation in Figure 9, the ELM probability peaks where the edge current perturbation is indeed positive, as expected. In the case of \# 20333, this is well after the voltage perturbation when the plasma recovers by moving vertically. For \#20334 and \#22768 it is on the leading edge of the perturbation.

The DINA simulations confirm the variation of the edge current as the plasma moves vertically, and also show the presence of a shorter edge current pulse in phase with the $\mathrm{G}$ coil current. The current induced by the motion is calculated by DINA to be of the same order of magnitude as the simple estimate proposed. The action of the closed feedback loop, the distortion of the magnetic surfaces and the inward diffusion of the driven edge current together provide a complex behaviour which might be simpler to study with modified perturbation waveforms, the object of future study on TCV.

\section{AN ILLUSTRATIVE MODEL OF MAGNETIC TRIGGERING}

Up to this point we have made the simple interpretation that positive perturbations in the edge current may destabilise MHD activity and thereby trigger an ELM. This is based on the observed correlation of the ELMs with vertical motion in the direction that acted to increase the edge current density. In these experiments, frequency entrainment was found to occur for driver frequencies both above and below the natural ELM frequency. To explain the latter, the perturbation must also periodically have a stabilising effect on the edge conditions, in order to cause a delay in the occurrence of the next ELM. This may be provided by the periodic reduction in edge current density caused by movement of the plasma in the opposite direction.

It is interesting to consider whether a simple model of the ELM cycle can reproduce any of the observations made during these experiments, such as frequency entrainment, and the intermittent losses in entrainment or "phase slipping". To this end we consider a simple way 
by which the edge current density $J$ and pressure gradient $\mathrm{p}^{\prime}$ at the H-mode pedestal may be dynamically linked. By including an abrupt drop in $\mathrm{p}^{\prime}$ when a boundary in $\left(\mathrm{p}^{\prime}, \mathrm{J}\right)$ space is exceeded, which symbolises the effect of crossing an ideal MHD stability threshold, relaxation oscillations representing ELMs are obtainable. The aim is to investigate the effect on these relaxation oscillations of adding a cyclic perturbation $\mathrm{J}_{\mathrm{ext}}$ to $\mathrm{J}$.

The first simplification made in the model is to consider a small layer of fixed width $\Delta \mathrm{x}$ just within the separatrix, so that $\mathrm{p}^{\prime}$ is simply given by $\mathrm{p} / \Delta \mathrm{x}$, where $\mathrm{p}$ is the pressure at the inner side of the layer. This allows $\mathrm{p}$ to be considered as a dynamical variable rather than its spatial derivative. The model equation for $\mathrm{p}$ is:

$$
\frac{\mathrm{dp}}{\mathrm{dt}}=\mathrm{Q}_{\mathrm{in}}(1+\sigma \varepsilon(\mathrm{t}))-\mathrm{W}(\mathrm{p}, \mathrm{J}) \mathrm{p}
$$

where $\mathrm{Q}_{\text {in }}$ represents the average power flux into the edge layer and $\sigma \varepsilon(\mathrm{t})$ represents fluctuations of amplitude $\sigma$ in the power flux. No attempt has been made to model these fluctuations self-consistently. Instead, we have tried different functions $\varepsilon(t)$ : either a superposition of several discrete frequencies or a Gaussian noise signal. As will be shown later, the results are not critically dependent on the model chosen for $\varepsilon(t)$. The function $W(p, J)$ is chosen proportional to an ideal MHD instability growth rate:

$$
\mathrm{W}(\mathrm{p}, \mathrm{J})=\frac{\mathrm{J}-\mathrm{J}_{\text {crit }}(\mathrm{p})}{\tau_{\mathrm{MHD}}}
$$

if $\mathrm{J}>\mathrm{J}_{\text {crit }}(\mathrm{p})$, and 0 otherwise. The function $\mathrm{J}_{\text {crit }}(\mathrm{p})$ parameterises the marginal stability boundary and is chosen be a parabolic function resembling that proposed by a detailed MHD analysis [4]. It is shown by the dotted curve in Figure 10a). The physical idea is that the triggering of an ideal MHD instability causes a loss of the energy content in the layer on a timescale of the order of ideal MHD phenomena, $\tau_{\mathrm{MHD}}$, which is a constant in the model.

The edge current density is modelled by:

$$
\frac{\mathrm{dJ}}{\mathrm{dt}}=\frac{\mathrm{J}_{0}(\mathrm{p})-\mathrm{J}+\mathrm{J}_{\mathrm{ext}}\left(\mathrm{f}_{\mathrm{D}}\right)}{\tau_{\mathrm{R}}}
$$

This equation simply models the return to steady state value $J_{o}(p)$ of $J$ over a timescale for current redistribution $\tau_{\mathrm{R}}$, which is a constant in the model, and where $\mathrm{J}_{\mathrm{ext}}$ is an external 
perturbation at a driving frequency $f_{D}$. The equilibrium current density $J_{o}$ is taken to be proportional to $\mathrm{p}^{\prime}$ (and therefore $\mathrm{p}$ in this model), which would be expected if the edge current was dominated by the bootstrap current.

When $\sigma$ and $J_{\text {ext }}$ are zero, the equilibrium values of pressure and current ( $p_{o}$ and $J_{0}$ ) may be found by setting the left hand sides of equations 4 and 6 to zero, and the dynamical stability of the equilibrium can be evaluated by standard means. Regular relaxation oscillations representing an ELM cycle are obtained when the equilibrium is made unstable, for example by increasing $\tau_{R}$ above a threshold value. Figure 10a) shows an example of a trajectory in $(p, J)$ space of a relaxation oscillation, or model ELM, computed numerically from the above equations. The ideal MHD stability threshold is marked by a dotted line in the figure, and the equilibrium curves for $\mathrm{dJ} / \mathrm{dt}=0$ and $\mathrm{dp} / \mathrm{dt}=0$ are marked by dashed lines. Figure $10 \mathrm{~b}$ ) shows the evolution of $J$ and $p$ as functions of time, starting from the initial condition $p=J=0.1$. The model ELM cycle in this case is characterised by a slow increase in $\mathrm{p}$ and $\mathrm{J}$ (with $\mathrm{J}$ lagging behind $\mathrm{p}$ because of the enforced time constant $\tau_{\mathrm{R}}$ ) until the ideal MHD stability threshold is reached, whereupon $\mathrm{p}$ rapidly collapses, producing the ELM event, until $\mathrm{p}$ is sufficiently low that the gains in equation 4 represented by $Q_{\text {in }}$ outweigh the losses $(\mathrm{Wp})$, allowing the cycle to repeat. The time of an ELM is defined by the point at which p reaches its peak value. The period of the relaxation oscillation was found to decrease smoothly with $\mathrm{Q}_{\text {in, }}$ a behaviour typical of type-I ELMs.

We show in Figure 10c) the time traces of $-d p / d t$ and $-d J / d t$ for an ELM cycle. The quantity $-\mathrm{dp} / \mathrm{dt}$ can be viewed as the energy flux out of the layer. It occurs in bursts which are reminiscent of the typical $\mathrm{D}_{\alpha}$ signals seen in the experiment. Increasing the fluctuation amplitude level $\sigma$ has the effect of increasing the level of random fluctuations in the ELM period around a mean value that is almost unchanged as compared to the $\sigma=0$ case.

We consider now a perturbation $\mathrm{J}_{\mathrm{ext}}=\mathrm{J}_{1} \cos \left(2 \pi \mathrm{f}_{\mathrm{D}} \mathrm{t}\right)$, with an amplitude $\mathrm{J}_{1}$ and a driving frequency $f_{D}=1 / \Delta t_{D}$ which is ramped slowly over a range that includes the average unperturbed ELM frequency $f_{\text {elm } 0}$, in a similar manner to the experiment. The results of the simulations are shown in Figure 11, on the left hand column for $\mathrm{J}_{1}=0.03$ and on the right hand column for $J_{1}=0.1$, with a fluctuation amplitude $\sigma=0.1$ for both. On the top row, Figure $11 \mathrm{a}$ ), are the time signals for $-\mathrm{dp} / \mathrm{dt}$; the second row, Figure $11 \mathrm{~b}$ ), shows the sequence of time intervals between consecutive ELMs, with the driver $\Delta t_{D}$ indicated by the dashed line and the 
unperturbed ELM repetition time, $\Delta \mathrm{t}_{\mathrm{elm} 0}=4.84$, by the dotted line; the third row, Figure 11c), contains the sequence of time delays between the external current perturbation and the ELM time; the fourth row, Figure 11d) shows the unwrapped phase between the driver signal and the ELM time plotted as a function of the driving frequency.

These results are to be compared with the experimental results of Figures 3 and 5. In spite of the simplicity of the model, the similarity with experimental measurements is striking. For small amplitude $\mathrm{J}_{1}=0.03$ (Figure 11 , left) there is hardly any effect of the perturbation on the ELM time sequence, which stays fluctuating around the unperturbed value (b). The time delays (c) and the phase (d) do not feel a visible effect either. The structure seen in the time delays (c) around $\mathrm{t}=500$ is simply due to the point in time when the driving frequency matches that of the unperturbed ELM. For a larger amplitude, $\mathrm{J}_{1}=0.1$ (right column), the effect of the current perturbation can be observed on all signals a)-d). There is a slight trend to decrease the average ELM amplitude as the driver frequency increases above the unperturbed ELM frequency, i.e. for $t>500$ (a). Between $t=350$ and $t=800$ the ELM sequence tends to synchronise with the driver perturbation with short time intervals for which the ELM times are seen to desynchronise. This is most clearly seen on the plots of time delays (c) and phase (d). For a driver frequency between 1 and 1.2 the phase exhibits phase "locking" intervals separated by phase "slipping" episodes. For a driving frequency between 1.2 and 1.7 the phase locks with small fluctuations, and for a driving frequency larger than 1.7 phase slipping events occur again. This behaviour is very similar to the phase slipping events found in the experiment (Figure 5).

We have considered several variations of the above model. First, replacing the parabolic stability boundary with straight lines in $(\mathrm{p}, \mathrm{J})$ space does not have a crucial effect on the behaviour shown in Figure 11. Second, we have considered a modified expression for the quantity $\mathrm{W}$ :

$W(p, J)=\left(\exp \left(\alpha_{B} p+\beta_{B} J-\gamma_{B}\right)+\exp \left(\alpha_{P} p+\beta_{P} J-\gamma_{P}\right)+1\right) / \tau_{E}$

This function takes a value close to $1 / \tau_{\mathrm{E}}$ (where $\tau_{\mathrm{E}}$ represents the energy confinement time between ELM events) when $\mathrm{p}$ and $\mathrm{J}$ are within the region of parameter space that is stable for ideal MHD ("Peeling- Ballooning") modes, and exponentially grows with J or $\mathrm{p}$ when the stability threshold is approached and crossed. The idea is to model a confinement degradation as the system moves towards the stability limits. Third, we have considered a Gaussian noise 
signal for $\varepsilon(\mathrm{t})$ instead of a superposition of sinusoidal signals. All these modifications were found to have a minor influence on the ELM time behaviour, and the conclusions drawn from Figure 11 still remain valid. Another interesting conclusion is that the ELM time sequence seems to be rather insensitive to details in the description of the ELM crash, for which the real physical processes are certainly quite complex.

Figure 12 shows the modelled ELM period and the driver period versus time in the left hand column, and the unwrapped phase with respect to the driver signal versus time in the right hand column, for three cases (the top, middle and bottom rows) in which $\sigma$ was set to $0,0.05$ and 0.1 respectively. In each case the model of Eq.(4), (6) and (7) and the same noise sequence $\varepsilon(\mathrm{t})$ were used.

The top example shows that the unperturbed ELM period $\Delta \mathrm{t}_{\mathrm{elm} 0}$ is about 5.2 in this case, and that the noise free response of the model to the perturbation can be classified into two groups as $\Delta t_{D}$ is varied. Firstly, asymmetric oscillations in $\Delta t_{\text {elm }}$ occur at a rate that decreases with $\left|\Delta \mathrm{t}_{\mathrm{elm} 0}-\Delta \mathrm{t}_{\mathrm{D}}\right|$, and corresponding step-like behaviour occurs in the phase for $\Delta \mathrm{t}_{\mathrm{D}}>6$ and $\Delta \mathrm{t}_{\mathrm{D}}<$ 4.6. Secondly, perfect synchronisation of the ELM period with the driver period occurs for 4.6 $<\Delta \mathrm{t}_{\mathrm{D}}<6$. The introduction of noise in the middle and bottom rows produces random variations in $\Delta \mathrm{t}_{\text {elm }}$ both with and without the perturbation, as expected. Although the precision of the synchronisation is reduced, the average $\Delta \mathrm{t}_{\mathrm{elm}}$ still follows the scan in $\Delta \mathrm{t}_{\mathrm{D}}$. It is interesting to note that the boundary between the two classes of behaviour described above becomes increasingly blurred as the noise level is increased. This has the effect of causing isolated events where $\Delta t_{\text {elm }}$ undergoes a rapid excursion from $\Delta t_{D}$ before returning, and the phase ramps through $2 \pi$ radians. This behaviour is again very similar to the phase slipping events found in the experiment, in particular to discharge \#20333. Specifically, isolated phase slipping events are found on either side of a period during which the phase is roughly flat (limited by the noise level) and $\Delta t_{\text {elm }}$ takes on a value close to $\Delta t_{D}$. The close repetition of phase slipping occurrences between discharges \#20333 and \#20334 however, suggests that the timing of these events are not determined by fluctuations due to noise. Of course we cannot exclude the existence of another non-linear model that produces similar behaviour in the absence of noise, in which irregularity in $\Delta \mathrm{t}_{\mathrm{elm}}$ is an intrinsic property of the model.

While neither of these models have a claim to be a full description of the ELM cycle, the finding of similar behaviour in the reaction of both experiment and model to external 
perturbations is significant, and provides some insight to the ELM dynamics. For example, the same classes of behaviour in the absence of noise are also found in classical non-linear models describing relaxation oscillations, such as the driven van der Pol oscillator. In this case, the asymmetric oscillations in the period are known as "periodic pulling" [13], and the phase and frequency variation may be described analytically. Our model results imply that this may be the root of the process leading to the phase slipping events seen in the experiments described.

\section{EXTRAPOLATION TO OTHER TOKAMAKS}

This new result indicating that it is possible to affect the triggering of ELMs raises two specific issues, namely the scaling to larger devices and scaling to tokamaks without a fast internal coil system.

To consider the extrapolation from a small to a larger device, we consider the simple case of identical tokamak geometries, expanded by a geometrical factor $\mathrm{K}_{\mathrm{G}}$. The toroidal magnetic field is independently increased by a factor $\mathrm{K}_{\mathrm{B}}$. The typical timescale for displacement of the plasma equilibrium is independently increased by a factor $\mathrm{K}_{\mathrm{T}}$, determined by the properties of the vertical position control system, the passive conducting structures and the PF coil power supplies. The electrical conductivity at the plasma edge is scaled by a factor $\mathrm{K}_{\mathrm{S}}$ as a result of changes to the underlying transport, probably dependent on the other factors. The following steps lead us directly to the change in the toroidal current at the plasma edge when the plasma moves by the same scaled distance in the vacuum vessel.

To obtain the same safety factor at the scaled toroidal magnetic field, the plasma current scales as $\mathrm{K}_{\mathrm{G}} \mathrm{K}_{\mathrm{B}}$. The PF currents, which produce the same vacuum flux pattern, scale with the plasma current. The values of the scaled flux contours scale as the vacuum currents divided by the scale lengths, multiplied by the scaled surface areas, namely as $\mathrm{K}_{\mathrm{G}}{ }^{2} \mathrm{~K}_{\mathrm{B}}$. If the plasma moves by a given scaled distance, then the flux change scales in the same way as the flux contours. The surface voltage is given by the change in flux divided by the timescale, and

therefore scales as $\mathrm{K}_{\mathrm{G}}{ }^{2} \mathrm{~K}_{\mathrm{B}} / \mathrm{K}_{\mathrm{T}}$. The surface electric field scales as the voltage divided by the circumference, namely as $\mathrm{K}_{\mathrm{G}} \mathrm{K}_{\mathrm{B}} / \mathrm{K}_{\mathrm{T}}$. The induced modulation of the current density at the edge of the plasma increases with the edge conductivity and the induced electric field variations, therefore it scales as $\mathrm{K}_{\mathrm{G}} \mathrm{K}_{\mathrm{B}} \mathrm{K}_{\mathrm{S}} / \mathrm{K}_{\mathrm{T}}$. For similar current profiles, the unperturbed current scales as $\mathrm{K}_{\mathrm{B}} / \mathrm{K}_{\mathrm{G}}$. The change in the edge current, normalised by the current on axis 
therefore scales as $\mathrm{K}_{\mathrm{G}}{ }^{2} \mathrm{~K}_{\mathrm{S}} / \mathrm{K}_{\mathrm{T}}$. This final result is consistent with a simple dimensional argument.

As an example, we scale from these TCV experiments to JET. $\mathrm{K}_{\mathrm{G}}$ is $2.9 / 0.88 \sim 3.5$. The pedestal electron temperature increases by roughly $2 \mathrm{keV} / 0.2 \mathrm{keV} \sim 10$, leading to $\mathrm{K}_{\mathrm{S}} \sim 30$. The magnetic field scales as $\mathrm{K}_{\mathrm{B}} \sim 3 / 1.5 \sim 2$. We assume that the timescale of the plasma movement increases with the square of the device size, as a first approximation, so $\mathrm{K}_{\mathrm{T}} \sim \mathrm{K}_{\mathrm{G}}{ }^{2}$ $\sim 10$. With these assumptions, we obtain the desired result that the edge current variation should scale by a factor of 36 .

This result is surprising at first sight, since the first guess is that the electromagnetics slow down with increasing device size, so the driving terms would slow down. However, the dominant term is the increase in the edge conductivity, since we have assumed no skin-effect for the diffusion of the flux and no inductive term in the impedance of the edge current, for simplicity. For TCV this seems to be a reasonable assumption, whereas for JET this might have to be examined more closely.

This encouraging result that the modulation of the edge current increases significantly for a larger tokamak naturally leads us to examine whether the particular feature used in these TCV experiments, namely the fast internal coils, would rule out this technique in tokamaks with only external PF coils. Generalising is more difficult, since the design of the control system for the vertical movement does not obey simple considerations like the ones used to scale the edge current modulation. The formulation proposed reduces the question to a simple one, namely how does the movement timescale scaling factor $\mathrm{K}_{\mathrm{T}}$ vary from device to device, especially when considering internal versus external control coils.

The speed of the vertical position closed loop depends on the vessel thickness and the power supply limits, which do not have a physical extrapolation, but do have clear tendencies. Without reviewing the design details, scaling from TCV to ITER gives a factor of $\mathrm{K}_{\mathrm{G}}{ }^{2} \mathrm{~K}_{\mathrm{s}} \sim$ $(6 / 0.88)^{2} \times(5 / 0.2) \sim 200$. The plasma vertical position in these TCV internal coil experiments has a timescale of $\sim 1 \mathrm{~ms}$ whereas the ITER control system should react in under 1 second. This implies that such an effect might be relevant even in ITER and that there is no fundamental adverse size scaling. 


\section{CONCLUSIONS}

An attempt to modify the ELM frequency has recently been made on TCV by applying a fast voltage perturbation to the radial field control coils during single null ELMy H-mode discharges. The input signal for the perturbation was added to the vertical stabilisation feedback loop, and consisted of a series of square pulses, which produced spike like pulse trains in the current through the radial field coil, and resulted in rapid deviations in the plasma vertical position. Phase synchronisation was found between the ELM cycle and the external perturbation. The ELM frequency was found to track scans in the external driver frequency about its unperturbed value over a frequency range that increased with the amplitude of the perturbation. It was also found that scaling of the ELM amplitude with the inter-ELM period was preserved in the presence of the perturbation, so that by altering the ELM period, we were also able to alter the ELM amplitude. DINA simulations showed that when phase locked, the ELMs were found to occur at times when the edge current density was increased under the action of the perturbation, either by direct induction from the changing current in the coil, or by movement of the plasma through the vacuum field. We therefore surmise that the effect of our perturbation is to modify the edge stability conditions by modulating the edge current, thereby acting to either delay or precipitate ELM events. Momentary interruptions in the phase lock were observed to occur during the driver frequency scan in a number of experiments.

Some of our observations suggest a prompt action by the perturbation, leading to an instantaneous crossing of the stability boundary governing ELM occurrences. For example, generating an edge current via a $\mathrm{G}$ coil pulse is shown to lead to a subsequent effect on the ELM occurrences, creating a peak in occurrence times after the beginning of the perturbation, as shown in Figure 6. Other features are reminiscent of a more continuous modification of the cyclic ELM behaviour in response to the perturbation. For example, it is difficult to explain by a prompt trigger model the observed synchronisation of the ELMs to a driver with a period that is longer than the natural ELM period, as in this case the ELMs must be delayed in order to remain synchronised. The DINA simulations show that the effect of the perturbation is felt during the whole inter-pulse period making a distinction between these two ideas difficult. New experiments with various pulse shapes are planned to resolve this question.

A simplistic dynamical model of the edge pressure gradient and current density that includes a threshold condition analogous to the ideal MHD stability boundary, was used to illustrate a 
mechanism by which ELMs could become phase locked under the action of current perturbations, and also how the observed momentary losses in phase lock may have occurred. The potential usefulness of an "ELM control" method based on this approach for ITER was examined by extrapolation from TCV using first approximation scaling arguments, and no fundamental adverse scaling effects were found.

\section{ACKNOWLEDGEMENTS}

The authors would like to thank Xavier Llobet and Simon Allfrey for many helpful discussions. We are grateful to the TCV team without whom these results would not be analysable. This work was partly supported by the fonds National Suisse de la Recherche Scientifique.

\section{REFERENCES}

[1] The ASDEX Team Nucl. Fusion 29 (1989)1959

[2] A. W. Degeling et al Plasma Phys. Control. Fusion 43 (2001) 1671

[3] A. Herrman. et al accepted for publication in Nucl. Materials.

[4] J. W. Connor et al Phys Plasmas 5 (1998) 2687

[5] H. R. Wilson et al Nuclear Fusion 40 (2000) 713

[6] S. J. Fielding et al Proc. $28^{\text {th }}$ EPS Conf. on Plasma Phys. and Control Fusion (Funchal) ECA 25A (2001) 1825-1828

[7] G. Saibene, private communication.

[8] P. T. Lang et al Proc. $29^{\text {th }}$ EPS Conf. on Plasma Phys. and Control. Fusion (Montreux) ECA 26B (2002) P1.044

[9] Y. R. Martin, A. W. Degeling and J. B. Lister, Plasma Phys. Control. Fusion, 44 (2002) A373

[10] R.R. Khayrutdinov and V.E. Lukash, J. Comp. Physics, 109 (1993) 193

[11] R.R. Khayrutdinov, J.B. Lister, V.E. Lukash and J.P. Wainwright, Plasma Phys.Control. Fusion, 43 (2001) 321

[12] J-Y. Favez, R. R. Khayrutdinov, J. B. Lister and V. E. Lukash, Plasma Phys. Control. Fusion, 44 (2002) 171

[13] T. Klinger et al Phys. Rev. E. 52 (1995) 4316 


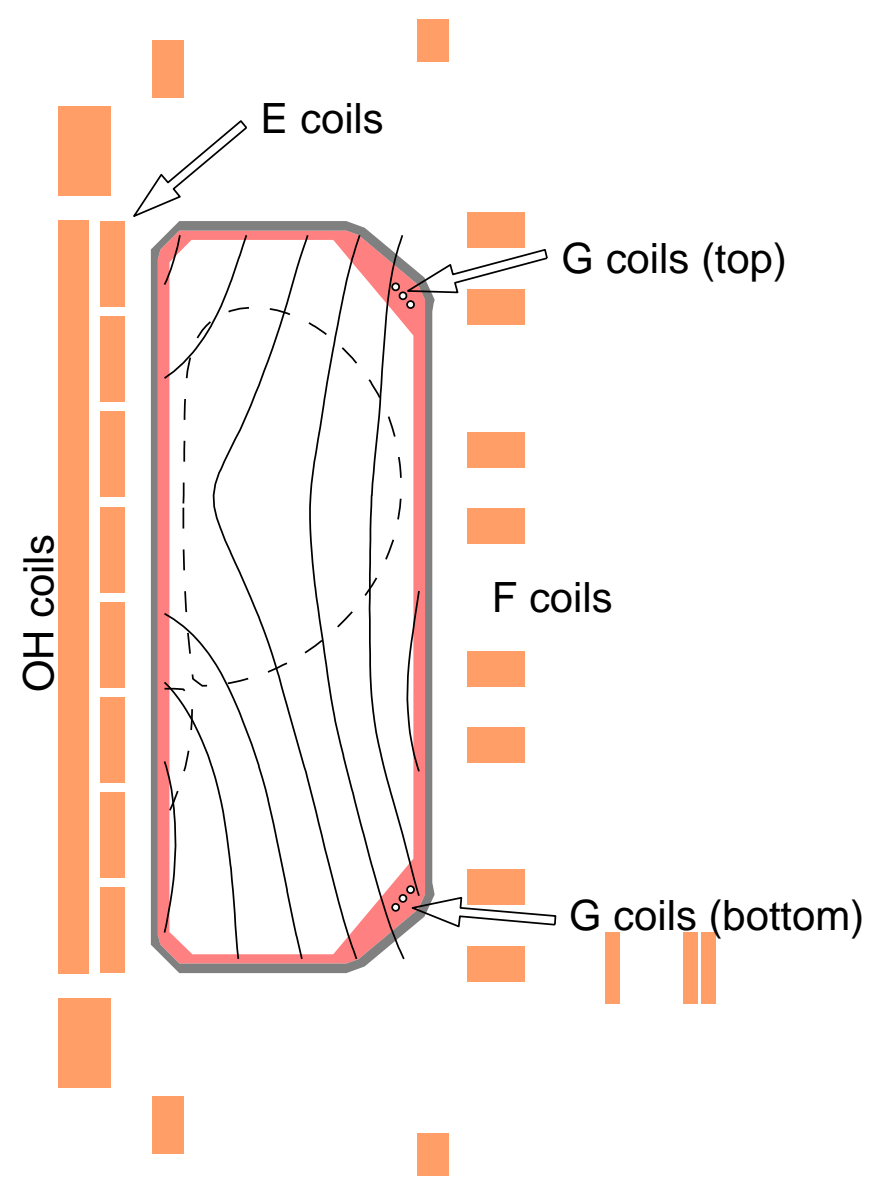

Figure 1: Cross section view of TCV vacuum vessel (dark grey), the carbon tiles (light grey) and poloidal field coils. The $G$ coils are located within the vacuum chamber at the top and bottom as shown. Also shown in the figure is the vacuum poloidal flux (solid lines) together with the separatrix (dashed line), both for discharge \#20333. 


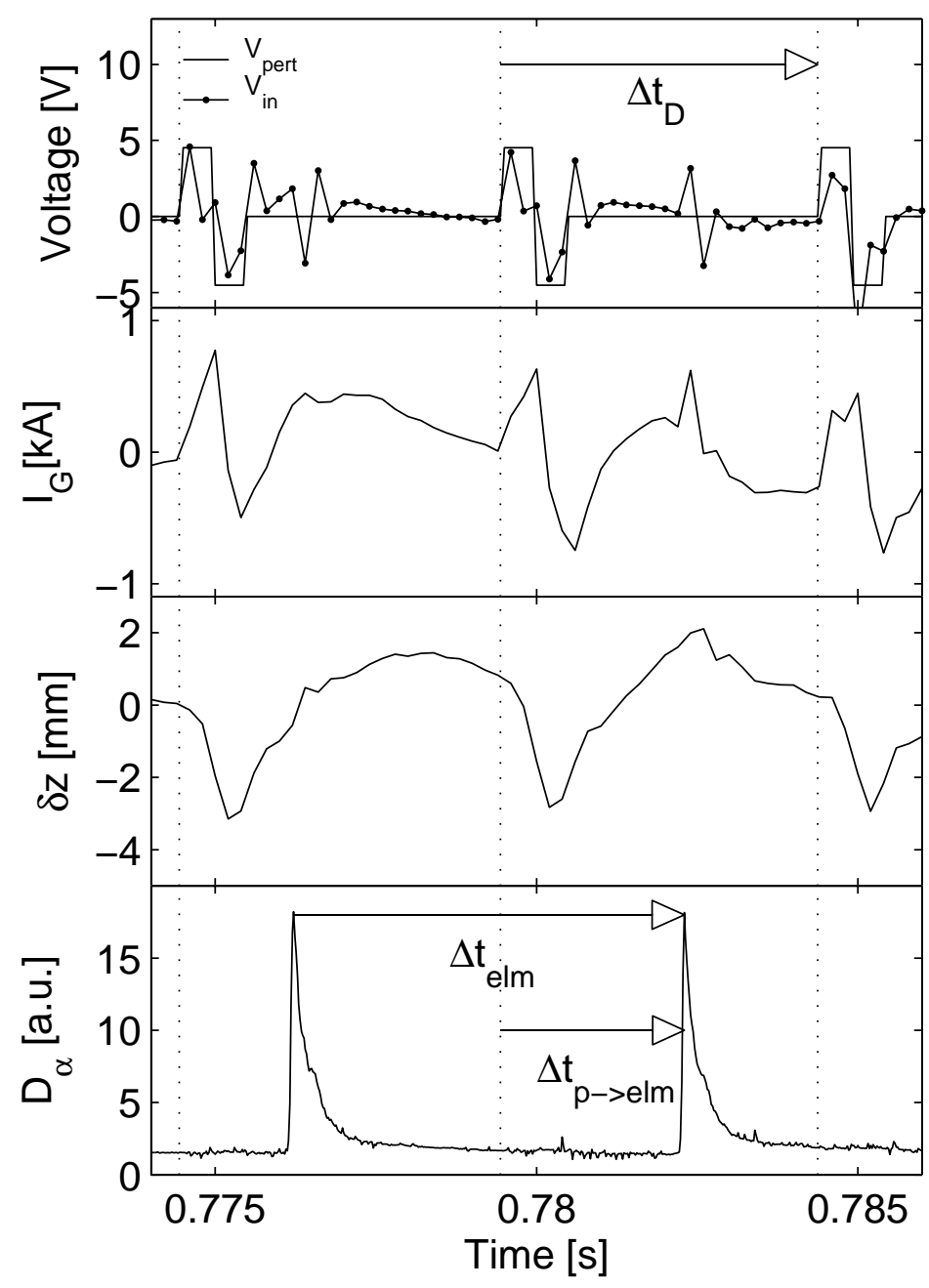

Figure 2: Detail of the perturbation input signals and the plasma response, showing the time variation of: a) The perturbation signal input to the plasma control system $\left(\mathrm{V}_{\text {pert }}\right)$ and the closed loop response $\left(V_{\text {in }}\right)$ which is input to the fast power supply for the $G$ coil; b) The G coil current $\left(\mathrm{I}_{\mathrm{G}}\right)$; c) The perturbed plasma position $(\delta \mathrm{z})$; d) The $\mathrm{D}_{\alpha}$ signal showing the occurrence of two ELMs. The definitions of the perturbation signal period $\left(\Delta t_{D}\right)$, the inter-ELM period $\left(\Delta \mathrm{t}_{\mathrm{elm}}\right)$ and the time delay from the perturbation to the next $\operatorname{ELM}\left(\Delta \mathrm{t}_{\mathrm{p} \rightarrow \mathrm{elm}}\right)$ are indicated by arrows on the diagram. 


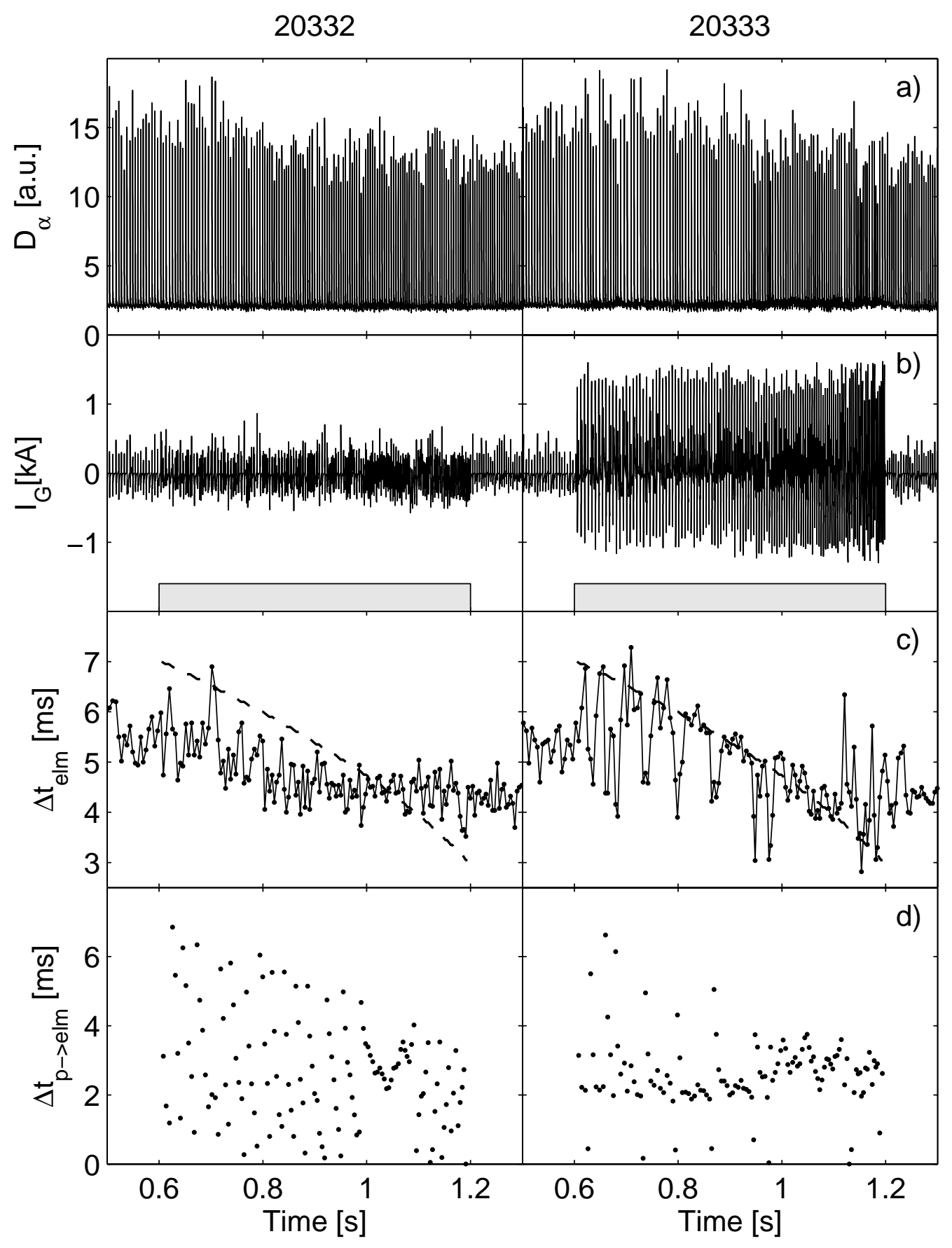

Figure 3: Comparison of discharges \#20332 and \#20333: a) $\mathrm{D}_{\alpha}$ signal showing ELM activity; b) $\mathrm{G}$ coil current, grey bar indicates the duration of the perturbations; c) Inter-ELM interval $\Delta \mathrm{t}_{\text {elm }}$ (points) and the drive period $\Delta \mathrm{t}_{\mathrm{D}}$ (dashed line); d) Time delay from each perturbation to the next ELM $\Delta \mathrm{t}_{\mathrm{p}->\mathrm{elm}}$. 


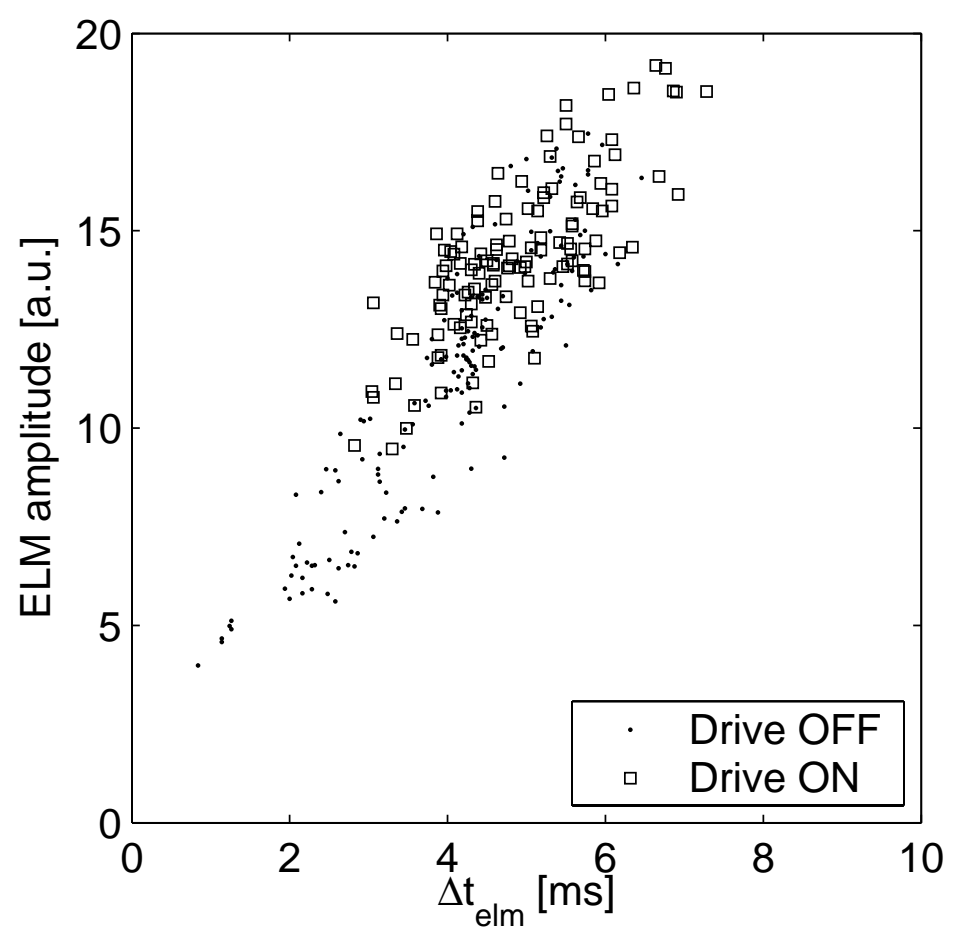

Figure 4: ELM amplitude (from $\mathrm{D}_{\alpha}$ signal) versus $\Delta \mathrm{t}_{\mathrm{elm}}$ for discharge \#20333. Squares indicate ELMs occurring while the perturbation was activated, and $\Delta t_{D}$ was swept from 7 to 3 ms. Dots indicate all other ELMs that occurred during the discharge, including the transient phases at the beginning and end of the H-mode. Note that the amplitude of ELMs both in the presence and absence of external perturbations have the same scaling with $\Delta \mathrm{t}_{\mathrm{elm}}$. 


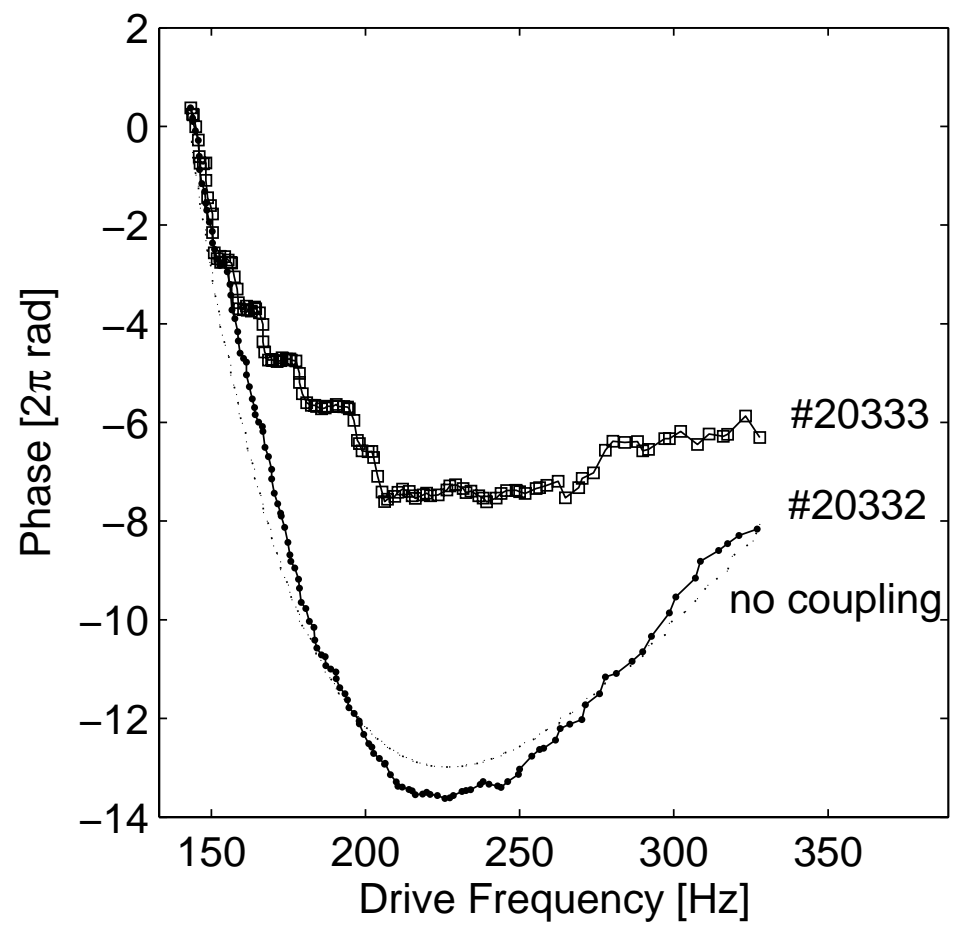

Figure 5: Comparison of the unwrapped phase of each ELM in the driver cycle as the drive frequency is swept in discharges \#20332 (points) and \#20333 (squares). The repeated step like features in discharge \#20333 indicate long periods when the ELMs were synchronised with the drive signal as its frequency was swept, interspersed by short periods where synchronisation was lost. No such features are evident in discharge \#20332, which follows the curve expected if no coupling existed (dotted line). 


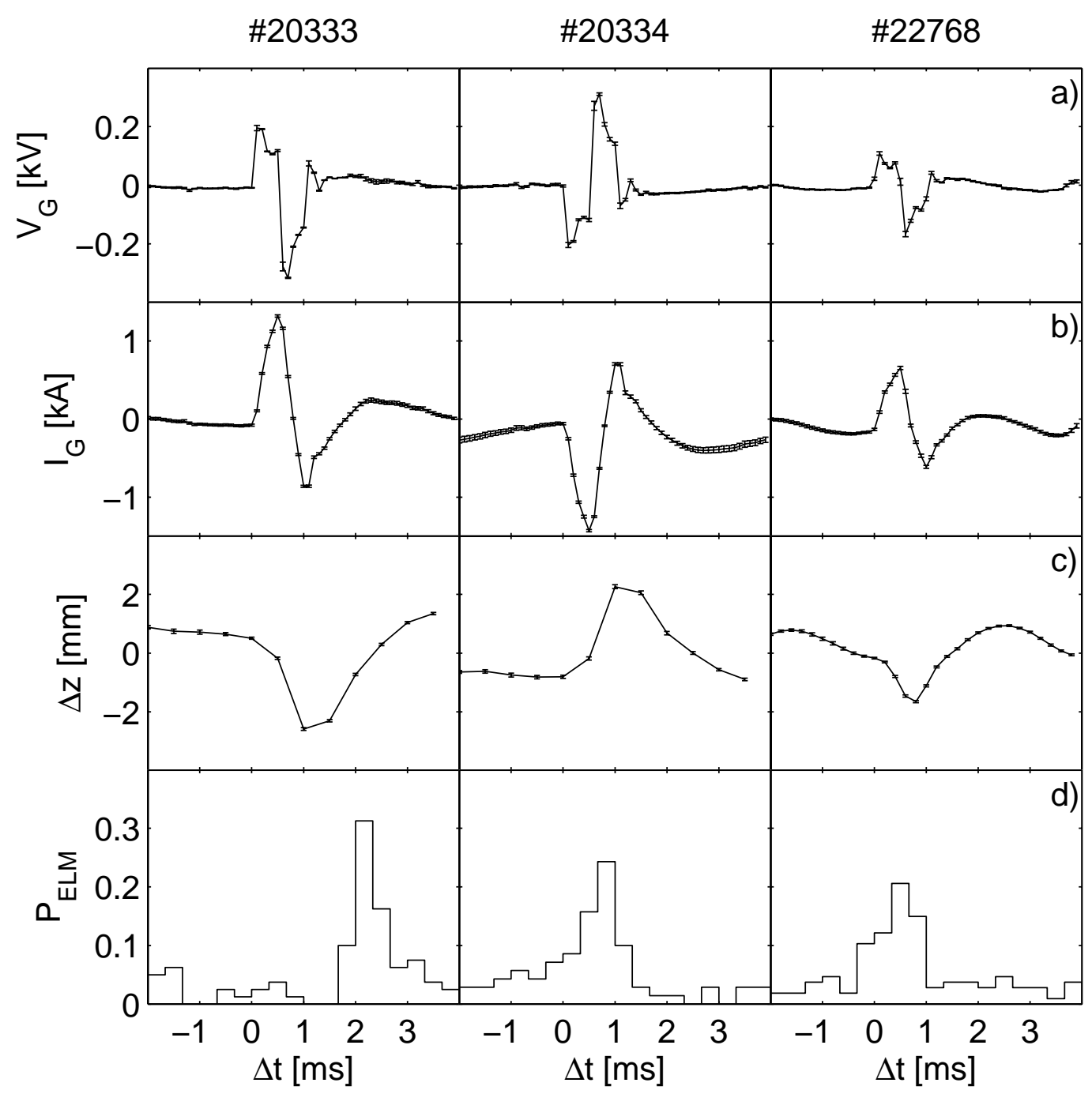

Figure 6: Comparison of perturbed parameters and the ELM occurrence probability for discharges \# 20333, \# 20334 and \# 22768: a - c) Phase coherent averages of the G coil voltage, current and perturbed vertical position as functions of time with respect to the perturbation input signal (where the perturbation onset occurs at $\Delta \mathrm{t}=0$ ); $\mathrm{d}$ ) ELM occurrence probability distribution, estimated from the histogram of time intervals to the first ELM before and after each pulse (based on about 100 ELMs in each case). 


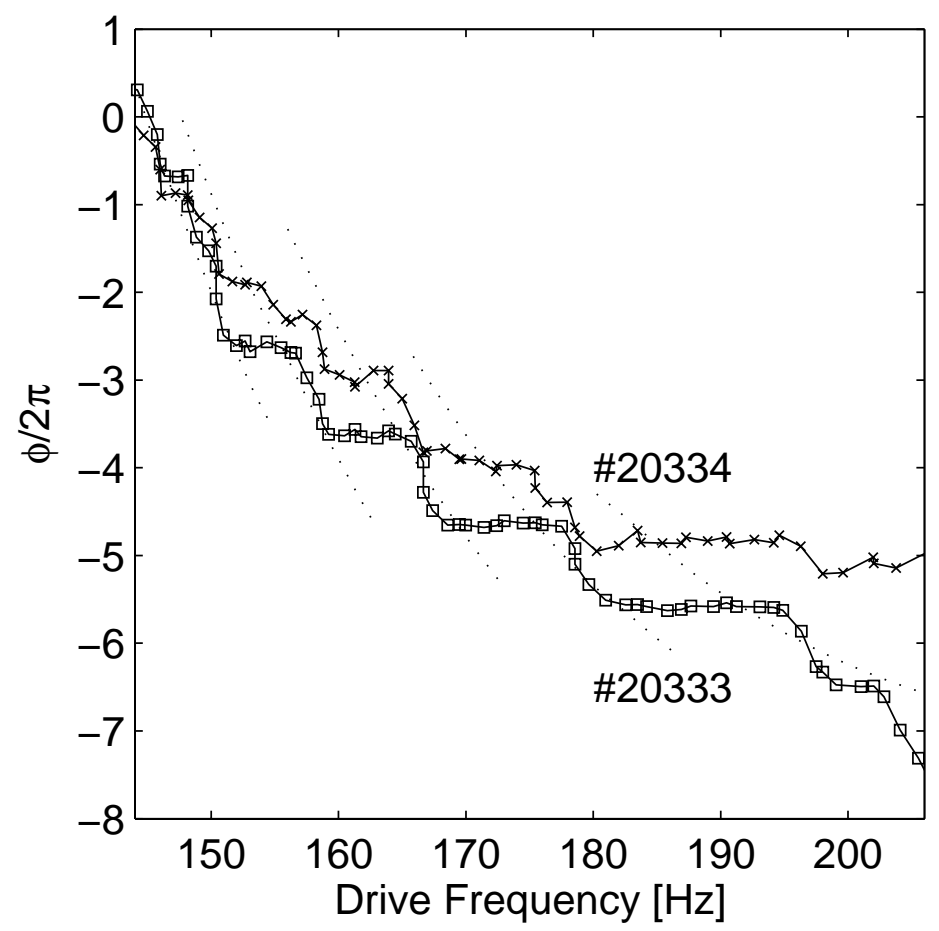

Figure 7: Detailed comparison of the unwrapped phase versus drive frequency for discharges \#20333 (squares) and \#20334 (crosses). The dotted line shows the phase variation expected if no coupling between ELMs and the driver existed during the episodes of phase slipping for discharge \#20333. Note that the instances of phase slipping are closely repeated in the two examples. 


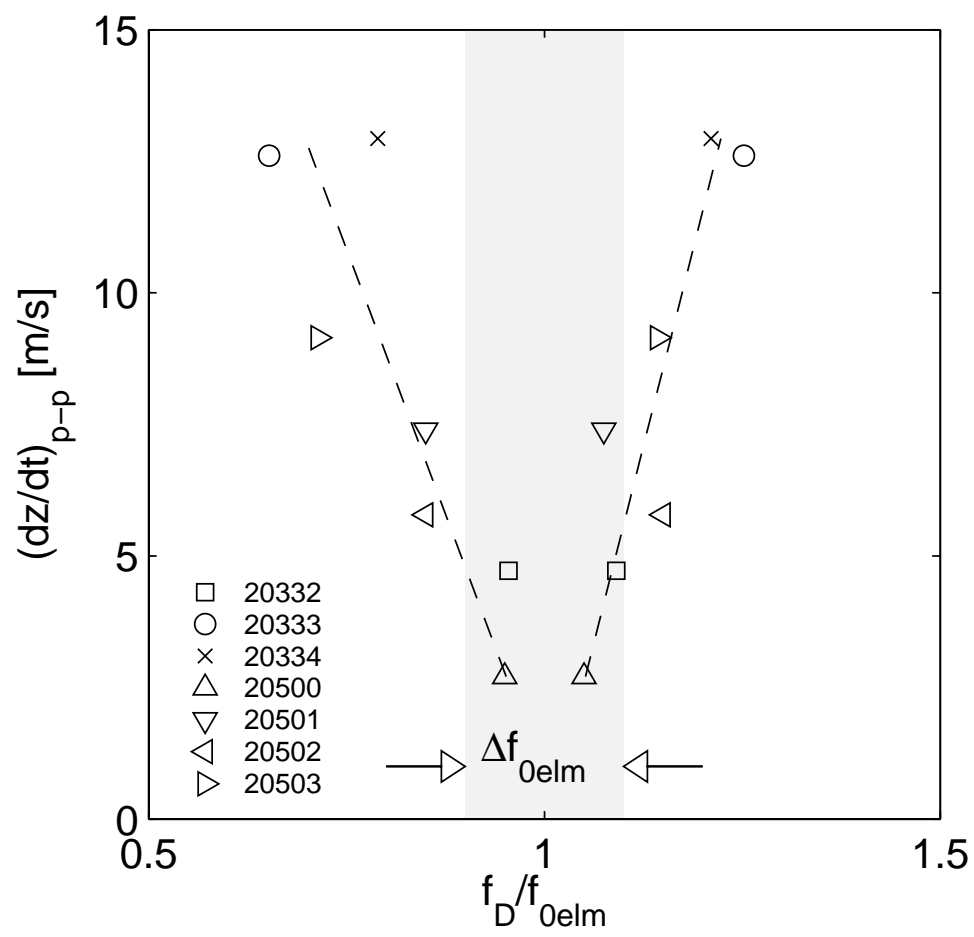

Figure 8: Estimated range in driver frequency (normalised by the average ELM frequency $\mathrm{f}_{0 \mathrm{elm}}$ during unperturbed periods of each discharge) over which the ELM frequency is affected by the applied perturbations, versus the peak-to-peak amplitude of the vertical plasma motion. The grey area represents the variation in the unperturbed ELM frequency $\left(\Delta \mathrm{f}_{0 \mathrm{elm}}\right)$. 


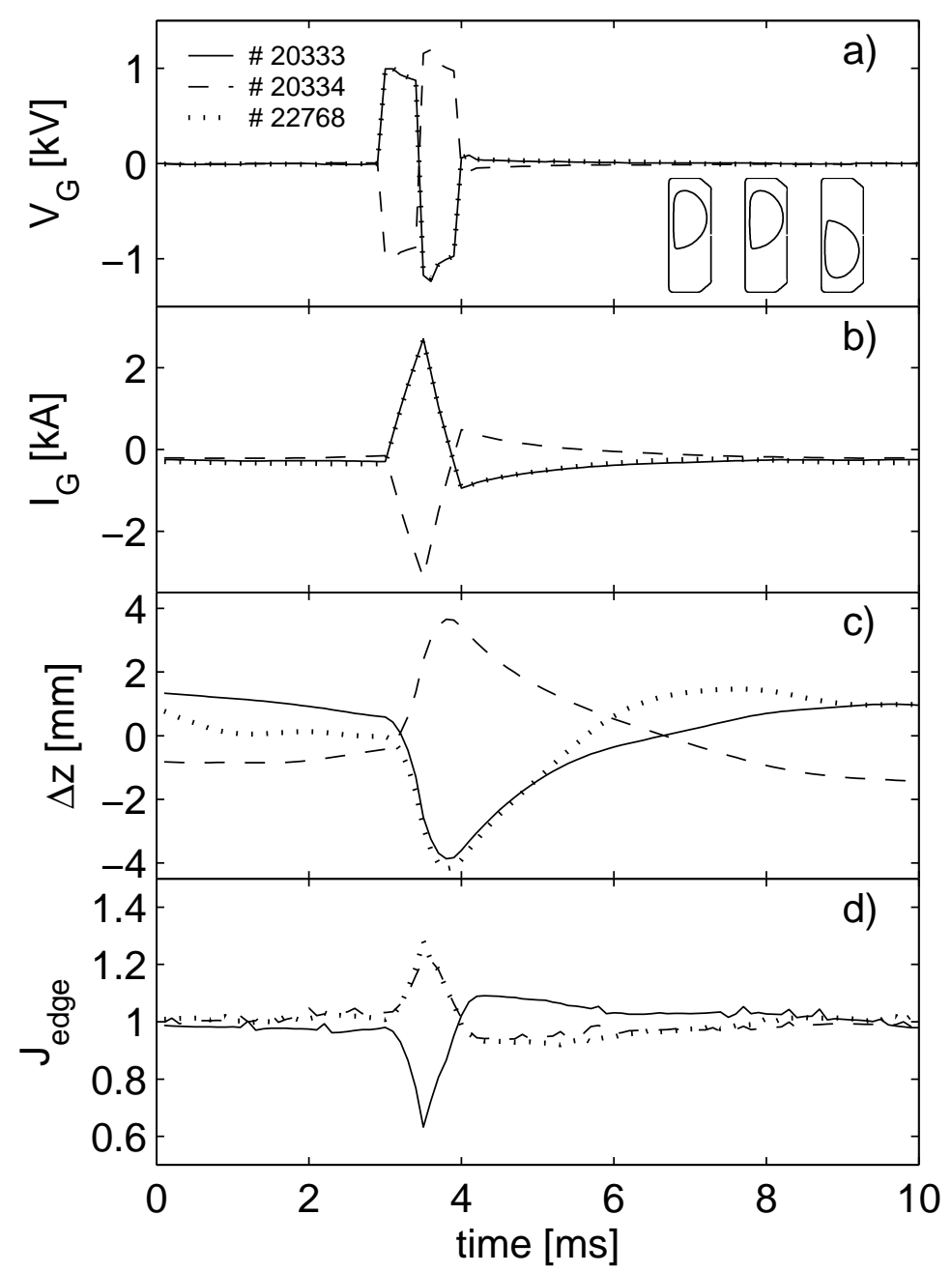

Figure 9: Result of 3 simulations of the G coil perturbation on three equilibria, shown in the inset (\#20333, \#20334, \#22768 from left to right). The vertical displacements are with respect to the mean vertical position. The edge current perturbations are shown normalised to the mean edge current. 

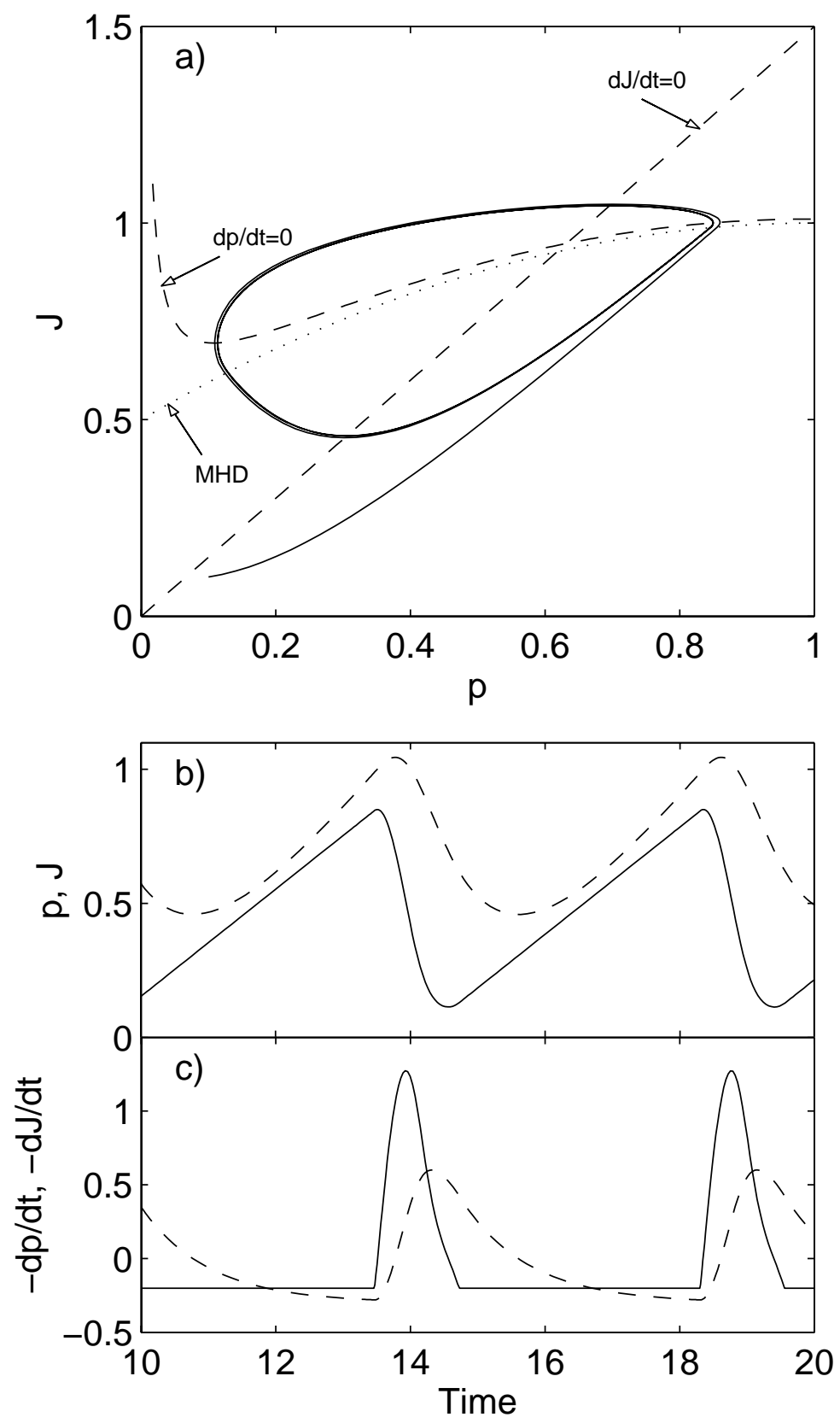

Figure 10: a) The solid line represents the evolution of trajectory in ( $p, J)$ space for the model of Eqs.(4)-(6). The dotted line, labelled MHD, represents the stability threshold. The equilibrium curves $\mathrm{dp} / \mathrm{dt}=0$ and $\mathrm{dJ} / \mathrm{dt}=0$ are indicated with dashed lines; $\mathrm{b}$ ) time evolution of $\mathrm{p}$ (solid line) and $\mathrm{J}$ (dashed line); c) time evolution of $-\mathrm{dp} / \mathrm{dt}$ (solid line) and $-\mathrm{dJ} / \mathrm{dt}$ (dashed line). 

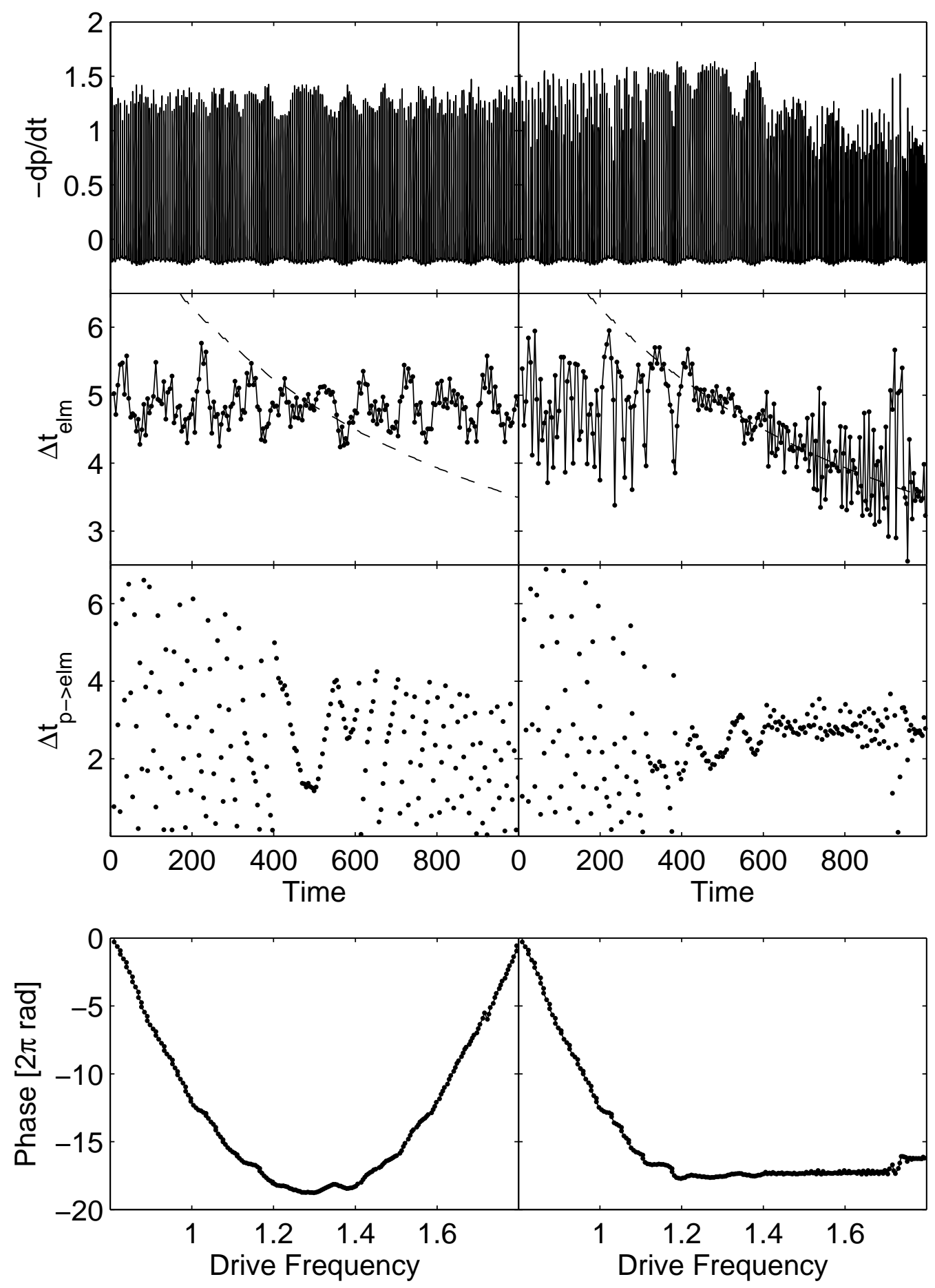

Figure 11: a)Time signals of -dp/dt; b) $\Delta \mathrm{t}_{\mathrm{elm}}$ (continuous line with dots), driver $\Delta \mathrm{t}_{\mathrm{D}}$ (dashed line) and unperturbed $\Delta \mathrm{t}_{\mathrm{elm} 0}$ (horizontal dotted line) c) time delay between current perturbation and ELM; d) unwrapped phase between current perturbation and ELM, for a small $\left(\mathrm{J}_{1}=0.03\right.$, left column $)$ and a large $\left(\mathrm{J}_{1}=0.1\right.$, right column $)$ current perturbation amplitude, using the model of Eqs (4)-(6) with a fluctuation amplitude $\sigma=0.1$. 


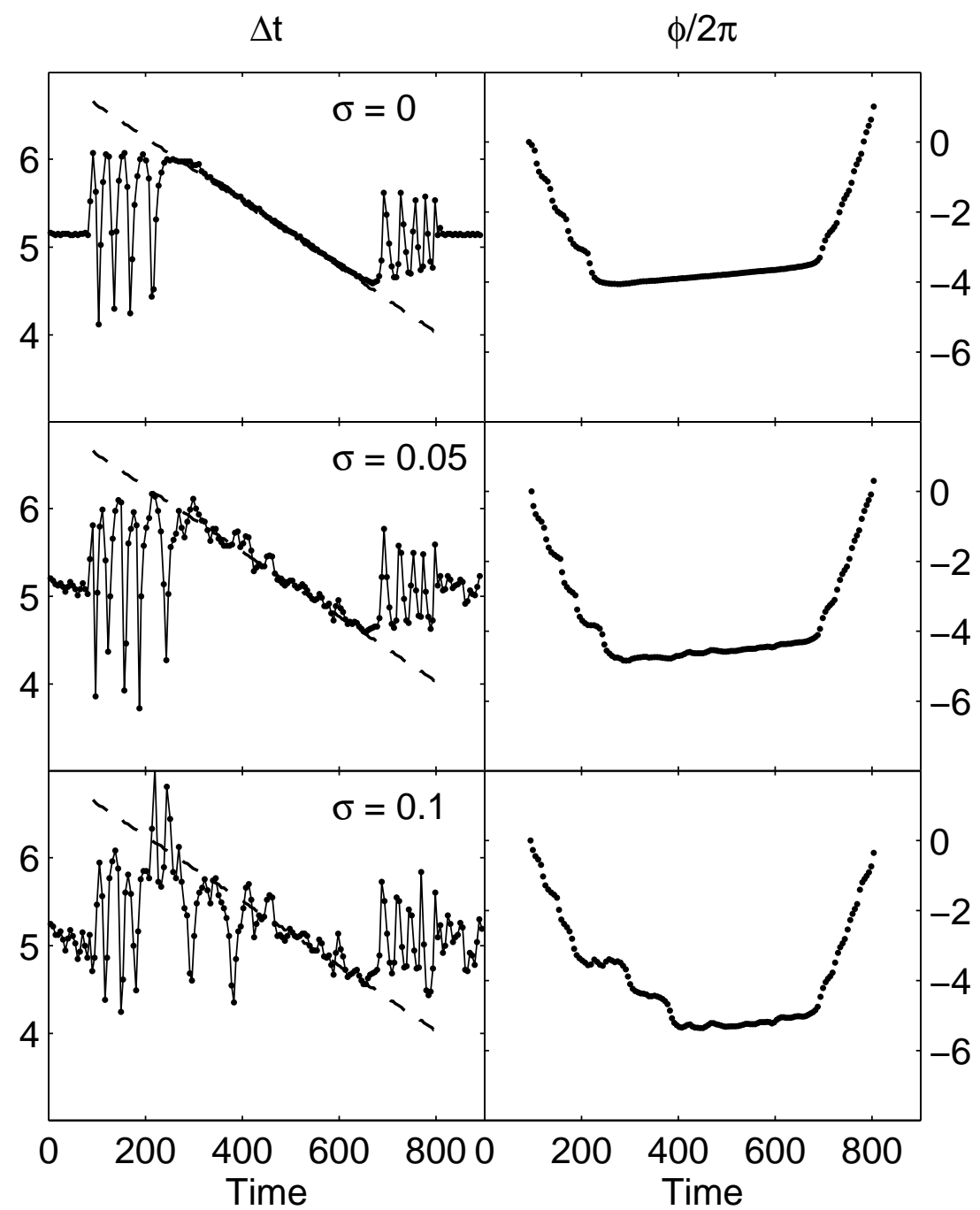

Figure 12: The response of the ELM dynamics to an external driver according to the model using eq. 7 for $\mathrm{W}(\mathrm{p}, \mathrm{J})$. Each row corresponds to an increasing noise level $(\sigma)$. Left column: ELM period (dots + solid line) and driver period (dashed line) as functions of time; Right column: ELM phase with respect to the driver as a function of time. 